\title{
An annotated list of the Pterophorinae (Oidaematophorini and Pterophorini) of Iran (Lepidoptera: Pterophoridae)
}

\author{
Helen Alipanah \& Peter Ustjuzhanin
}

Alipanah, H. \& Ustjuzhanin, P. 2005: An annotated list of the Pterophorinae (Oidaematophorini and Pterophorini) of Iran (Lepidoptera: Pterophoridae). Entomol. Fennica 16: 129-143.

A checklist of the 29 species of Pterophorinae (tribus Oidaematophorini and Pterophorini) of Iran is presented. Calyciphora golestanica sp. n., and the hitherto unknown male of Merrifieldia farsi (Arenberger 1981) and the female of Tabulaephorus djebeli (Arenberger 1981) are described. Emmelina argoteles (Meyrick 1922) and Hellinsia pectodactylus (Staudinger 1859), are reported as new for Iran. The known distribution of each species is given.

H. Alipanah, Insect Taxonomy Research Department, Plant Pests and Diseases Research Institute, P.O. Box 19395-1454, Tehran, Iran; E-mail: alipanah @ppdri.ac.ir

P. Ustjuzhanin, Siberian Division of the Russian Entomological Society, clo Zoological Institute, Academy of Science, Universitetskaya naberezhnaja 1, 199034 St.Petersburg, Russia; E-mail: alucita@online.sinor.ru

Received 9 November 2004, accepted 17 January 2005

\section{Introduction}

The pterophorid moths of Iran have not been studied in detail and only a few publications (e.g. Lederer 1869, 1870, Zerny 1940, Amsel 1959, Bigot 1968, Arenberger 1981, 1995a, 2002) are available. The Pterophorinae Zeller, 1841 is a large subfamily of which 112 species (belonging to the tribuses Oidaematophorini and Pterophorini) are known from the Palaearctic region (Arenberger 1995b). The tribuses together comprise more than 430 species, of which most occur in the New World and Afrotropical regions (Gielis 2003).

Iran is highly interesting from a zoogeographic point of view, owing to its geographic position and geological diversity (Fig. 1). Here not only do faunal elements of Asia Minor, the Middle East and Central Asia meet, but a rich en- demic fauna and flora has also evolved. As a consequence, Iran has always been of high interest as far as field research is concerned (Kallies \& Spatenka 2003). Golestan National Park, the locality of the type-species here described, is a "living museum" that includes various plants and animals. It is located within three provinces, viz. Golestan, Khorasan and Semnan.

Our aim in this paper is to revise the published information and other new data based on the original investigations. While examining the pterophorine material, collected from different parts of Iran, 29 species were identified.

\section{Material and methods}

This study was mostly based on the material preserved in the following collections: Hayk Mir- 
zayans Insect Museum (HMIM) of Plant Pests and Diseases Research Institute of Iran (PPDRI), National Natuurhistorisch Museum (Naturalis), Leiden, the Netherlands, and Zoological Institute of the Russian Academy of Science (ZIN), St. Petersburg, Russia. The summarized data from the literature are given in "Records from Iran", additional data are listed under "Material examined".

\section{Results}

\subsection{Tribus Pterophorini}

Pterophorus pentadactyla (Linnaeus, 1758)

Phalaena pentadactyla Linnaeus, 1758, Syst. Nat. (Edn. 10) 1: 542.

Phalaena tridactyla Scopoli, 1763 (nec Linnaeus, 1758), Ent. Carniolica: 257.

Alucita pentadactyla [Denis \& Schiffermüller], 1775, Ankündung Syst. Werkes Schmett. Wienergegend: 146.

Alucita pentadactyla Hübner, [1805], Samml. Eur. Schmett. 9: taf. 1, fig. 1.

Aciptilia pentadactyla var. sulphurea Staudinger, 1880, Horae Soc. Ent. Ross. 15 (1879): 433.

Material examined. 1 ô, Māzandarān (Chālakrud, Ekrāsar), 1,560 m a.s.1., 11.IX.1990, E. Ebrahimi \& M. Badii leg.; 1 q, Golestān (Golestān National Pārk, Tang-e Gol), 620 m a.s.1., 23-25.V. 1986, A. Pazuki leg., 1 đ̋ 2 우, (Golestān National Pārk, Kārkandeh), 5.VII. 2003, H. Rajai leg.; 1 q, Māzandarān (Tonekābon), 11.VII.1974, M. Abai leg.; 1 +, Gilān (Asālem, Shondol), 950 m a.s.1., 9.VII.1974, H. Mirzayans \& Gh. Farahbakhsh leg.; 1 đ̃, Gilān (Asālem, Pisāson), 750 m a.s.1., 13.VIII.1974, H. Mirzayans leg.1 +, Pisāson, 1,300 m a.s.1., 16-19.VIII.1980, A. Pazuki \& H. Broumand leg.; 1 , West Āzarbāijān (Orumieh, Sizvand Mt.), 24.VI.1970, A. Pazuki leg.

Records from Iran. Nowshahr, Tālesh, Hājiabād (Arenberger 1995b).

Distribution. Palaearctic region, excluding southern parts and Japan.

Remarks. The Iranian specimens are similar to those illustrated by Arenberger (1995b), but

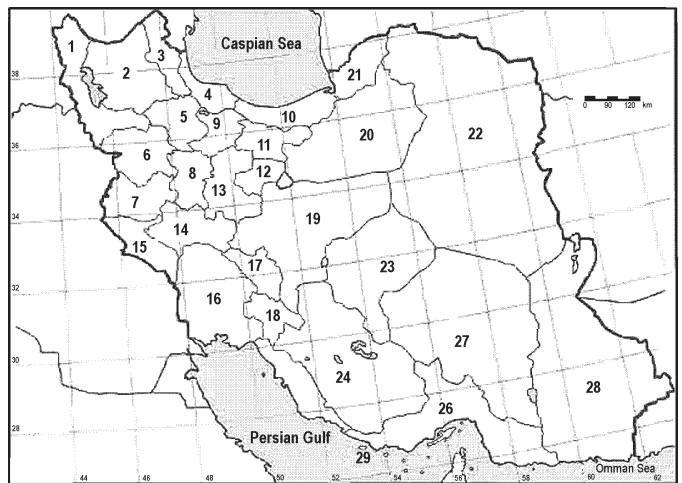

Fig. 1. Provinces of Iran. 1 - Āzarbāijān-e Gharbi; 2 Āzarbāijān-e Sharghi; 3 - Ardabil; 4 - Gilān; 5 - Zanjān; 6 - Kordestān; 7 - Kermānshāh; 8 - Hamedān; 9 - Ghazvin; 10 - Māzandarān; 11 - Tehrān; 12 - Ghom; 13 - Markazi; 14 - Lorestān; 15 - Ilām; 16 - Khuzestān; 17 - Chāhārmahāl \& Bakhtiāri; 18 - Kohgiluyeh \& Boyerahmad; 19 - Esfahān; 20 - Semnān; 21 - Golestān; 22 - Khorāsān; 23 - Yazd; 24 - Fārs; 25 - Bushehr; 26 - Hormozgān; 27 - Kermān; 28 - Sistān \& Baluchestān.

the wingspan of the examined specimens is 20-26 $\mathrm{mm}(n=8)$, while the normal range is 21-30 mm. This species is restricted to the north and north-west of Iran.

Pterophorus ischnodactyla (Treitschke, 1835)

Alucita ischnodactyla Treitschke, 1835, [in] Ochsenheimer, Schmett. Eur. 10 (3): 223.

Aciptilia actinodactyla Chrétien, 1891, Naturaliste 13 (2): 99.

Aciptilia eburnella Amsel, 1968, Stuttg. Beitr. Naturk. 191: 14, 15.

Records from Iran. Iran; Zagulajev (1986).

Distribution. Mediterranean region; northward to the Balkans and Slovakia; Pakistan and outer territories of the eastern Palaearctic region; Afrotropical, Republic of South Africa.

\section{Merrifieldia malacodactylus (Zeller, 1847)}

Pterophorus malacodactylus Zeller, 1847, Isis Oken, Leipzig 10 (12): 905-906.

Pterophorus meristodactylus Zeller, 1852, Linn. Ent. 6: 396.

Aciptilia baliodactylus var. meridionalis Staudinger, 1880, Horae. Soc. Ent. Ross. 15: 432. 
Alucita indocta Meyrick, 1913, Exot. Microlep. 1: 111.

Alucita subtilis Caradja, 1920, Dt. Ent. Z. Iria 34: 81 .

Alucita parca Meyrick, 1921, Exot. Microlep. 2: 421.

Alucita subcretosa Meyrick, 1922, Exot. Microlep. 2: 549.

Alucita spicidactyla Chrétien, 1923, Amat. Papillons 1: 231.

Alucita phaeoschista Meyrick, 1923, Entomologist 56: 277.

Alucita spicidactyla Chrétien, 1923, Amat. Papillons 1: 229-235.

Alucita luteodactyla Turati, 1926, Atti. Soc.

Ital. Sci. nat. 65: 66, 67.

Alucita rayatella Amsel, 1959, Bull. Soc. Ent.

Egypte 43: 55-56.

Aciptilla spicidactyla insularis Bigot, 1961, Lambillionea 61 (7-8): 49-51.

Aciptilia livadiensis Zagulajev \& Filippova, 1976, Trudy Zool. Inst. Leninger. 64: 40-41.

Pterophorus malacodactylus ssp. transdanubinus Fazekas, 1986, Ent. Z. Frankfurt a. M. 96 (1/2): 13-15.

Merrifieldia garrigae Bigot \& Picard, 1989, Alexanor 15 (7) 1988 (1989), Suppl.: [33]-[34]. Merrifieldia moulignieri Nel, 1991, Alexanor 17 (3): 144-178.

Merrifieldia inopinata Bigot, Nel \& Picard, 1993, Alexanor 18 (22): 122-128.

Material examined. $4 \hat{\jmath} \hat{\jmath}$, West Āzarbāijān (Orumieh, 15 km S), 2.IV.1975, M. Abai leg.; 1 , Golestān (Golestān National Park, Dasht-e Shād), 1,390 m a.s.1., 25-26.VII.1995, E. Ebrahimi \& V. Nazari leg.; 1 đ̃, Kermān, Baft-Jiroft (Shingera), 2,800 m a.s.1., 22-23.V.2004, H.

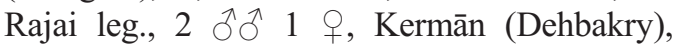
2,400-2,600 m a.s.1., 21-22.V.2004, H. Rajai leg.

Records from Iran. 3 ๙ิฮ 2 우, Alborz, (Karaj), 1937; 1 ô, Fārs, (Shirāz-Kāzerun road, Sineh Sefid), 2,200 m a.s.l., 5. VI.1937 (Bigot 1968); 1 †, Fārs, environs of Shiraz, 1,600 m a.s.1., 15.IV.1937, leg. Brandt., $1 \jmath^{\jmath} 1$ q , Karaj, Alborz Mt., 10.VI.1936, 1,800 m a.s.l., W. Brandt leg. (Arenberger 1981): Karaj (Alborz Mt.), Shirāz (Arenberger 1995b).

Distribution. Mediterranean region, eastward to Central Asia.
Remarks. In the male genitalia of the Iranian specimens, the shape of the valve is somewhat different from that illustrated by Arenberger (1995b): The left valve is broad with a blunt tip and the right valve nearly lanceolate with a moderately acute tip; harpe similar to that of the Tultscha specimen. Uncus longer, without acute tip (Fig. 2a). In one of the examined specimens the wingspan is $21 \mathrm{~mm}$, while the normal range is $10-20 \mathrm{~mm}$.

\section{Merrifieldia caspius (Lederer, 1870)}

Pterophorus caspius Lederer, 1870, Horae Soc. Ent. Ross. 8: 27 (1870).

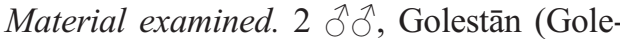
stān National Pārk, Ālmeh), 1,700 m a.s.l., 25.VII.2001, E. Gilasian, M. Mofidi-Neyestanak \& R. Ghayurfar leg.; 5 q, , Golestān (Golestān National Pārk, Sulgerd), 1,150 m a.s.l., 20-21.VII.1996, E. Ebrahimi \& V. Nazari leg.

Records from Iran. N E Iran (Toll, 1947); 4 đ̂̉, Khorāsān, (Binālud Mt., Mashhad), 1,800 m a.s.1., 15.VII-15.VIII.1938, 1 q, Fārs, (Shirāz-Kāzerun road, Sineh Sefid), 2,200 m a.s.l., 28.V.1937 (Bigot 1968); Khorāsān, Binālud Mt. (Mashhad), $1800 \mathrm{~m}$ a.s.1., 15.VIII.1938, leg. Brandt; Hyrcania, Mirābi Mt., 2,000 m a.s.1., VII-VIII (Arenberger 1981); Gorgān, Fārs, Mirābi Mt., Binālud Mt., Mashhad (Arenberger 1995b).

Distribution. Turkey, Iran, Turkmenistan, Uzbekistan, Kyrgyzstan, India, China.

Remarks. The examined specimens are somewhat smaller (wingspan of $18-21 \mathrm{~mm}, n=7$ ) than those reported by Arenberger (1995b). In the male genitalia, harpe of the left valva is straight and the right valva is not curved on the dorsal margin (Fig. 2b).

Merrifieldia brandti (Arenberger, 1981)

Pterophorus brandti Arenberger, 1981, Z ArbGem. Öst. Ent. 32(3/4) 1980 (1981): 107.

Records from Iran. 1 ô, S. Iran, 4-7.VI.1969, Miyān Kotal, 1,900 m a.s.1., E. Kāzerun, H. G. Amsel leg.; 2 ठึ่ 4 우, S. Iran, 4-7.VI.1969, Miyān Kotal, 1,900 m a.s.1., E. Kāzerun, H. G. Amsel leg.; 1 ō, S. Iran, Fārs, Kāzerun, MiyānKotal, 1,900 m a.s.1., 11.VI.1972, Ebert \& 


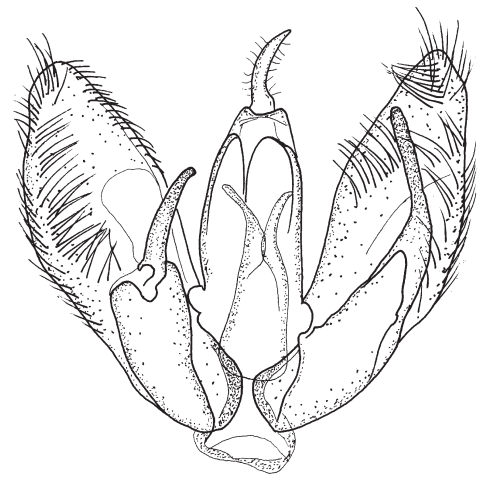

a
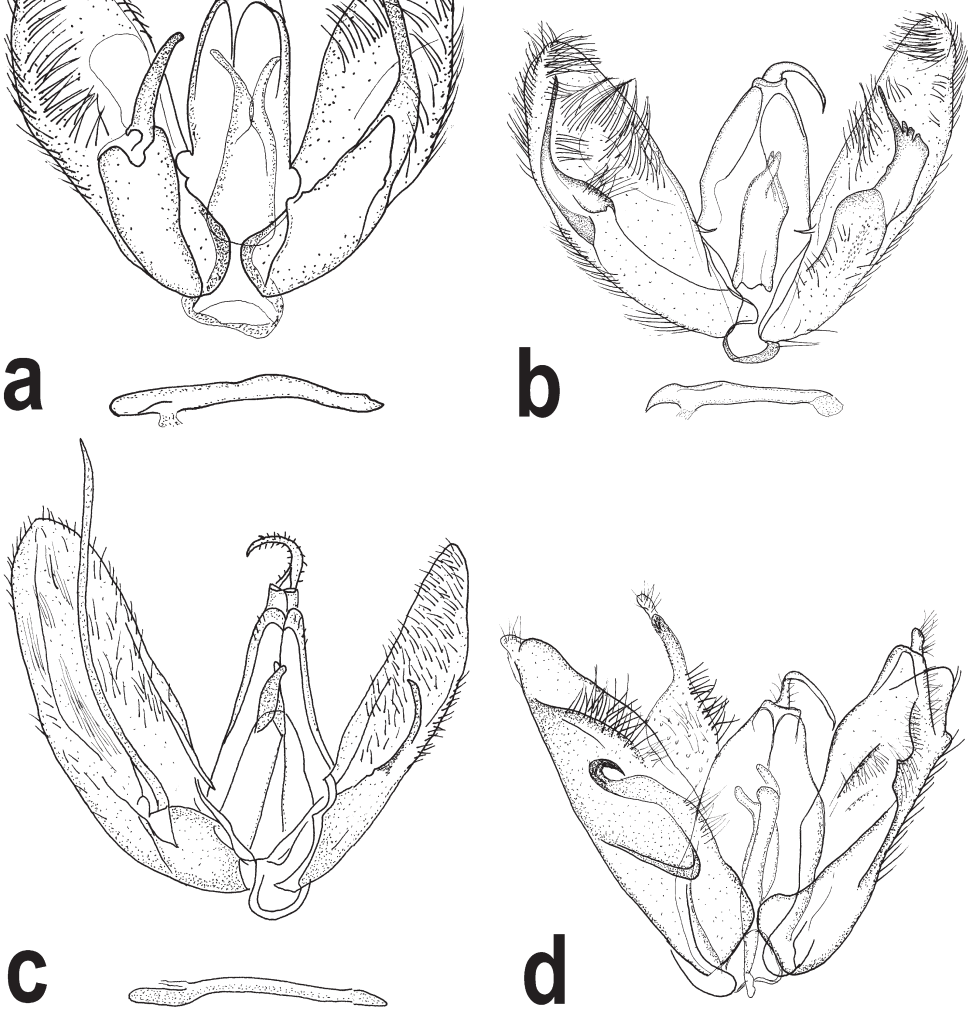

C

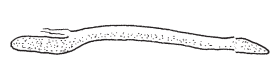

Fig.2. Male genitalia. - a. Merrifieldia malacodactyla (Zeller). - b. Male genitalia of Merrifieldia caspia (Lederer). - c. Merrifieldia farsi (Arenberger). - d. Emmelina argoteles (Meyrick).
Falkner leg.; 1 đ̃, Alborz-Mt., (Karaj), 1936, leg. W. Brandt; 3 ふ̄ ô, Fārs (Shirāz-Kāzerun road, Sineh Sefid), 2,200 m a.s.1., 28.V.1937, leg. Brandt (Arenberger 1981); Karaj (Alborz Mt.) (Arenberger 1995b).

Distribution. Turkey, Iran.

\section{Merrifieldia tridactyla ( Linnaéus, 1758)}

Phalaena tridactyla Linnaeus, 1758, Syst. Nat. (Edn 10) 1: 542.

Pterophorus fuscolimbatus Duponchel, 1844, Hist. Nat. Lépid. Papillion Fr. (Suppl.) 4: 498.

Pterophorus icterodactylus Mann, 1855, Verh. Zool.-bot. Ges. Wien 5: 571.

Alucita icterodactyla ab. noctis Caradja, 1920, Dt. Ent. Z. Iris 34: 83.

Alucita spicidactyla var. flavella Chrétien, 1923, Amat. Papillons 1: 232..

Alucita baliodactyla $f$. menthae Chrétien, 1925, Amat. Papillons 2: 243.
Alucita icerodactyla phillipsi Huggins, 1955, Entomologist's Gaz. 6: 124-126.

Aciptilia exilidactyla Buszko, 1975, Polskie pismo Ent. 45: 141-146.

Merrifieldia neli Bigot \& Picard, 1989, Alexanor 15 (7), 1988 (1989), Suppl.: [31]-[33].

Material examined. 1 Oे, West Āzarbāijān (W. Māku, Ghezelbulāgh), 27.VII.1976, A. Pazuki \& H. Broumand leg.; 1 \%, Kordestān (45km. S W Saghez), 2,000 m a.s.1., 30.VI.1975, A. Pazuki leg.

Records from Iran. Alvand (Arenberger 1995b).

Distribution. Ireland, England, France, Spain, Portugal, Morocco, Algeria, Malta, Italy, Corsica, Sardinia, Germany, Denmark, Sweden, Estonia, Poland, Chechnya, Slovakia, Austria, Hungary, Croatia, Yugoslavia, Bulgaria, Greece, Turkey, Russia, Kazakhstan, Iran.

Remarks. Specimens of both sexes have a narrow brownish band on the costal margin of the 


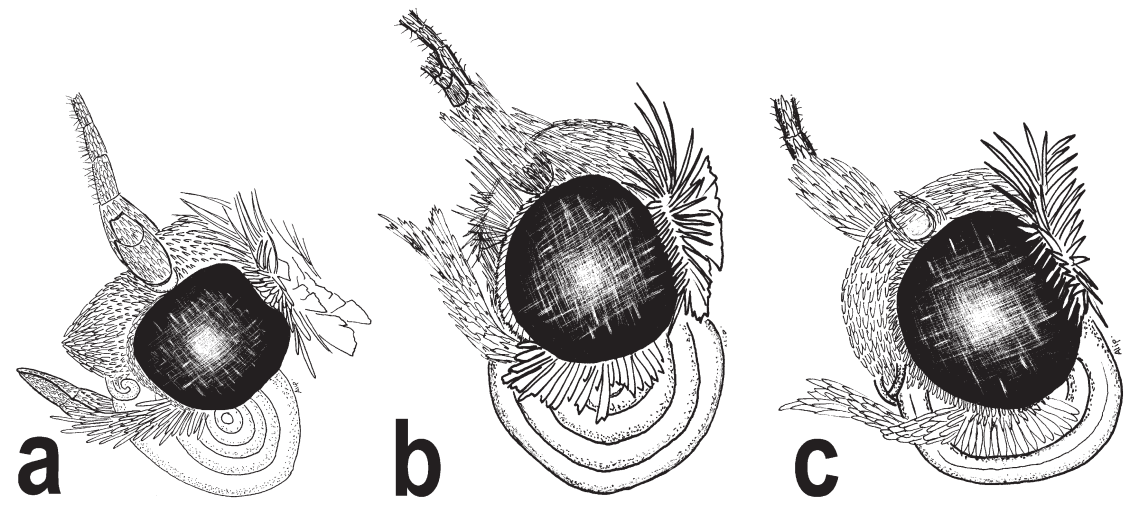

Fig.3. - Heads. - a. Merrifieldia farsi (Arenberger), male (Iran: Fars, Firuzabad, Muk, 8-9.V.1985, H. Mirzayans \& A. Hashemi leg.). - b. Tabulaephorus djebeli (Arenberger), female [Iran: Golestān (Golestān National Pārk, Ālmeh), 1.IX.1987, A Pazuki leg.]. - c. Calyciphora golestanica sp. n., female [Iran: Golestān (Golestān National Pārk, Ālmeh), 1,650m a.s.I., 17-25.V.1988, A. Pazuki leg.].

forewing, which extends to the top of the first lobe. The male genitalia of examined specimens are very similar to those from Morocco.

\section{Merrifieldia calcarius (Lederer, 1870)}

Pterophorus calcarius Lederer, 1870, Horae Soc. Ent. Ross. 8: 26 (1870).

Material examined. 2 우, Golestān (Golestān National Park, Yākhti Kālān), 2.VII.1985, A. Pazuki leg.

Records from Iran. NE Iran (Toll, 1947); 4

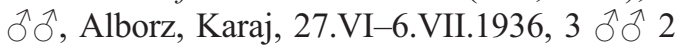
우, Fārs (Shiraz-Kāzerun road, Sineh Sefid), 2,200 m a.s.1., V-VII.1937 (Bigot, 1968); Gorgān, (Shāhkuh Mt., Hājiābād) (Arenberger 1995b).

Distribution. Iran, Turkmenistan, Kazakhstan.

Remarks. The colour and size of the examined specimens compare well to those illustrated by Arenberger (1995b).

\section{Merrifieldia farsi (Arenberger, 1981)}

Pterophorus farsi Arenberger, 1981, Z. ArbGem. Öst. Ent. 33(1/2): 22, 23.

Material examined. 1 đ̊, Fārs (ābādeh: $3 \mathrm{~km} \mathrm{~S}$ Didegān), 1,900 m a.s.1., 20.VI.1972, G. Ebert \& A. Pazuki leg.; 1 , Fārs (38 km S Ābādeh, Rudān), 270 m a.s.1., 29.IV.1986, H. Mirzayans
\& A. Hashemi leg.; 1 q, Fārs (Shirāz, Dasht-e Arjan), 1,940 m a.s.1., 6.VI.1990, E. Ebrahimi \& M. Badii leg.; 1 đ 1 ㅇ, Fārs (Firuzābād, Muk), 1,800 m a.s.1., 8-9.V.1985, H. Mirzayans \& A. Hashemi leg.

Remarks. M. farsi was described from $10 \mathrm{fe-}$ males collected by Ebert and Falkner from Abadeh (3 km S. of Didegan, Fars Province in southern part of Iran on 20.VI.1972); the male had not been described. Along with the females, there was an unknown male specimen having the same collecting data. Since the unknown male specimen did not match any other species and was very similar to the female of $M$. farsi, it was considered as male of $M$. farsi.

Description. Wingspan $24 \mathrm{~mm}$. Head with scales appressed, creamy-white; collar with some erect scales; frons prominent, somewhat pointed at apex (Fig. 3a). Palps curved upward, white with some erect scales at base of segment I and underside of segment II. Antenna white dorsally and brownish ventrally; shortly ciliated ventrally and laterally. Thorax creamy white. Abdomen white, with a dark median longitudinal line dorsally. The examined male specimen is very similar to the female in having dirty white ground colour on the forewings. On the upperside of the forewing at the base of the first lobe, there is a faint brownish area. Fringes creamywhite with a brownish area medially on upperside of first lobe at middle. Ventrally, there is a small, faint, striped 
brownish area near the base of the first lobe, and another subapically at the first and second lobes.

Male genitalia (Fig.2c). Valves asymmetrical; right valve narrow, apex more or less pointed; left valve rounded, longer and twice as broad as the right one. Harpe (saccular process) of left valve very long, extending beyond valve, more than $1 / 5$ length of valve. Harpe of right valve short, more or less bent toward costa; about 1/5 length of left harpe. Anellus arms short. Uncus curved, as long as right harpe. Aedeagus straight, without cornuti.

Diagnosis. Considering the shape of the aedeagus and harpe on right valve in the male genitalia, Merrifieldia farsi somewhat resembles Merrifieldia cana Arenberger, 1990, but differs from that, as well as from other species, in having a very long and peculiar shape of the harpe on the left valve, extending beyond the valve apex; it also differs by the coloration of the wings and differences in the female genitalia.

Distribution. Iran.

\section{Merrifieldia diwani (Arenberger, 1981)}

Pterophorus diwani Arenberger, 1981, Z. ArbGem. Öst. Ent. 32(3/4) 1980 (1981): 101-102.

Material examined. 1 , Zanjān-Bijār $(50 \mathrm{~km}$ SW Zanjān), 1,800 m a.s.1., 28.VI.1975, A. Pazuki leg.

Records from Iran. 1 đ̂, Fārs, FF.18, Tangāb-e Firuzābād, 6.IV.1950, E. P. Wiltshire leg.; 1 †, S Iran, 4-7.VI.1969, Miyān Kotal, 1,900 m a.s.1., E. Kāzerun, H. G. Amsel leg. (Arenberger 1981); Fārs (Kāzerun) (Arenberger 1995b).

Distribution. Iran, Turkey.

Remarks. In the female genitalia, the signa are not curved.

\section{Wheeleria elbursi (Arenberger, 1981)}

Pterophorus elbursi Arenberger, 1981, Z. ArbGem. Öst. Ent. 32(3/4) 1980 (1981): 105-106.

Material examined. 4 ๙ิ $\widehat{\jmath}$, Tehrān (Karaj, Arangeh), 1,550 m a.s.1., 15.VI.1972, H. Mirzayans, M. Abai, M. Kavian \& M. Ghāziof leg.; 2 ふ̂̉, West Āzarbāijān (15km Bāzargān), 1,450 m a.s.1., 5.VI.1975, M. Abai leg.; 4 ठิ઼ 2
우, Gilān (Rudbār, Bivarzin), 900 m a.s.l., 27-28.V.1997, A. M. Sarafrazi, M. Badii \& V. Nazari leg.

Records from Iran. 2 Ђิ 6 우, N Iran, Alborz Mt., 12km Karaj, 1,650 m a.s.1., 12.VI. 1969, H. G. Amsel leg., 1 đ, N.W. Iran, 17 km. N W Māku, 1,400 m a.s.1., 4.VI.1975, H. G. Amsel

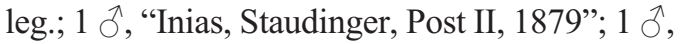
N Iran, 27.V.1975, 10 km N Karaj, Holyschuh \& Ressl leg.; 1 đ̃, Kermān (Esfandagheh), 24.IV. 1971, M. Safavi and M. Zairi leg.; 2 시 2 우우, Karaj, Alborz Mt., 1936, W. Brandt leg., 1 ๙, Fārs (Shirāz-Kāzerun road, Sineh Sefid), 2,200 m a.s.1., 5.VI.1937, Brandt leg. (Arenberger, 1981); Karaj (Alborz Mt.), Māku, Chālus, Esfandagheh, Fārs (Kāzerun) (Arenberger 1995b).

Distribution. Iran, Turkey.

\section{Wheeleria kabuli (Arenberger, 1981)}

Pterophorus kabuli Arenberger, 1981, Z. ArbGem. Öst. Ent. 33(1/2): 20, 21.

Material examined. 1 +, Māzandarān (Nur, Baladeh), 21.IX.1987, A. Hashemi leg.

Records from Iran. Alborz Mt., Polur (Arenberger 1995b).

Distribution. Afghanistan, Iran.

\section{Wheeleria sobeidae (Arenberger, 1981)}

Pterophorus sobeidae Arenberger, 1981, Z. ArbGem. Öst. Ent. 32(3/4) 1980 (1981): 103-105.

Material examined. 1 $\hat{\jmath}$, Ghom, $(8 \mathrm{~km} \mathrm{~S}$ Fordu, Vesb), 2,320 m a.s.1., 7-8.VI.1984, A. Pazuki \& A. Hashemi leg.; 1 $\hat{\jmath}$, Kohgiluyeh and Boyerahmad (Yāsuj, Sisakht), 2,100 m a.s.1., 16.VI.1986, H. Mirzayans \& A. Hashemi leg.

Records from Iran. 3 $\widehat{\jmath}$, N Iran, Alborz Mt., southern parts $30 \mathrm{~km} \mathrm{~N}$ Tehrān (before Shemshak), 27.V.1971, C. Holzschuh leg.; 1 đึ 2 우, N Iran, Alborz Mt., southern parts, $30 \mathrm{~km} \mathrm{~N}$ Tehrān (before Shemshak), 26.V.1971, C. Holzschuh leg.; 3 ふ઼઼, N Iran, 1,600 m a.s.1., Alborz Mt., Polur, 21.VI.1969, H. G. Amsel leg.; 2 우, N Iran, Alborz Mt., Māzandarān, Polur, Damāvand, 2,500 m a.s.1., 7-10.VII.1972, Ebert \& Falkner leg.; 1 ô, S Iran, Kohgiluyeh and Boyerahmad, Yāsuj, Sisakht, 2,250 m a.s.1., 13-14.VI.1972, Ebert \& Falkner leg.; 2 ๙ิ के, S Iran, Kohgiluyeh and Boyerahmad, $30 \mathrm{~km} \mathrm{~S}$ 
Yāsuj, Kushk, 2200 m a.s.1., 12.VI.1972, Ebert \& Falkner leg.; 1 ô, Baluchestān, Nikshahr, Tange Sarheh, 1,100 m a.s.1., 16.V.1972, Ebert \& Falkner leg.; 1 ô, 7-15.VI.1973, Darband, $25 \mathrm{~km}$ N Tehrān, 2,000 m a.s.1., Kasy \& Vartjan leg.; 3 ふ̄ đ̂, 28-30.V.1963, Darband, 25 km N Tehrān, 2,000 $\mathrm{m}$ a.s.1., Kasy \& Vartjan leg. (Arenberger 1981); Alborz Mts, Shemshak, Polur, Darband, Karaj, Nikshahr (Arenberger 1995b); 1 ๙ૈ, Esfahān (Kāshān, 40 km S Jahagh), 2,000 m a.s.1., 14-15.VI.1984, A. Pazuki \& A. Hashemi leg.; 1 đ̂ 1 q, Lorestān (Aznā, Darband), 1,700 m a.s.1., 5-6.VI.2000, M. Badii \& M. Mofidi-Neyestanak leg.

Distribution. Iran, Afghanistan, Turkey.

Remarks. The examined specimens are identical to those illustrated by Arenberger (1995b).

\section{Wheeleria spilodactyla (Curtis, 1827)}

Pterophorus spilodactylus Curtis, 1827, Brit. Ent. 4: folio 161.

Aciptilus xerodactylus Herrich-Schaffer, 1855, nec Zeller, 1841, Syst. Bearbeitung Schmett. Eur. 5: 384.

Aciptilia tuneta Staudinger, 1892, Dt. Ent. Z. Iris 5: 300.

Alucita calamodactyla Zerny, 1935, Mem. Soc. Sci. nat. phys. Maroc. 42: 129-130.

Records from Iran. Reineh-Damāvand (2,600-2,700 m a.s.1.), 20-27.VII.; Kandovan (2,800 m a.s.1.), 3-8.VII., 1 ô, Polur (2,000 m a.s.1.), 18-28.VII (Zerny 1940); 1 ते 3 우우, Alborz, Karaj, VIII-IX.1936, 1 ○ 1 ㅇ, Alborz, Nesā (Bigot 1968).

Distribution. England, France, Spain, Morocco, Algeria, Tunisia, Libya, Corsica, Sardinia, Italy, Belgium, The Netherlands, Germany, Poland, Slovakia, Ukraine, Iran.

\section{Wheeleria obsoletus (Zeller, 1841)}

Pterophorus obsoletus Zeller, 1841, Isis Oken, Leipzig, 1841: 859-860.

Aciptilus confusus Herrich-Schaffer, 1855, Syst. Bearbeitung Schmett. Eur. 5: 384.

Alucita desertorum Zeller, 1867, Stett. Ent. Ztg 28: 386.

Pterophorus subalternans Lederer, 1870, Horae Soc. Ent. Ross. 6: 93 (partim).
Alucita gonoscia Meyrick, 1922, Exot. Microlepid. 2: 549.

Alucita phlomidactyla Wasserthal, 1970, Z. Morph. Tiere 68: 213, 214.

Alucita marrubii Wasserthal, 1970, Z. Morph. Tiere 68: 214, 215.

Material examined. 1 q, Tehrān (Evin), 12.VII.1967, H. Mirzayans leg.; 1 đ, Tehrān (Disin), 17.VIII.1973, G. Ebert leg.; 1 đે, Tehrān (Tāleghān), 26-31.VII.1976, M. Kavian leg.; 1 옹 Tehrān (Tāleghān, Tijkuh), 1,950 m a.s.1., 9.VII.1997, M. Mofidi-Neyestanak \& H. Barari leg.; 2 우, Tehrān (6 km E Āzadbar-Tāleghān), 2,350 m a.s.1., 21.VII.1988, H. Mirzayans \& M. Badii leg.; 1 ô, Tehrān (Shahrestānak, Cheshmeh-e Kiā Kolā), 2,400 m a.s.1., 20.VII. 1988, H. Mirzayans \& M. Badii leg., 1 q and 1 , Tehrān (Karaj, Kandavān, Sarchāl), 2,800 m a.s.1., 4-8.VII.1977, A. Pazuki \& A. Mortazaviha leg.; 1 ô, Golestān (Golestān National Pārk, Ālmeh), 1,600 m a.s.1., 20.V.1974, A. Pazuki leg.; 2 đ̄ô, Fārs (Kāzerun, Nowdān), 1,250 m a.s.1., 17.V.1975, M Abai \& A. Pazuki leg.; 1 q, Fārs (ābādeh, Didegān), 2,000 m a.s.l., 20.V.1974, A. Pazuki \& M. Abai leg.; 1 đ̃, Fārs (Shirāz, Sivand), 1,150 m a.s.1., 15.V.1974, M. Abai \& A. Pazuki leg.; 1 đ̃, Semnān (Shāhrud, Kālpush), 1,300 m a.s.1., 25-26.VIII.1982, A. Hashemi leg.; $2 \widehat{\jmath} \widehat{\partial}$, Ardebil (Sareyn, Ardestān), 1,700 $\mathrm{m}$ a.s.1., 2.VII.1997, M. MofidiNeyestanak \& H. Barari leg.; 1 q, Esfahān (Kāshān, Ghohrud, Jewinān), 2,000 m a.s.1., 12-13.VI.1984, A. Pazuki \& A. Hashemi leg.; 3 우, West Āzarbāijān (Māku, Ghezelbulāgh), 1,910 m a.s.1., 27.VIII.1976 A. Pazuki \& H.

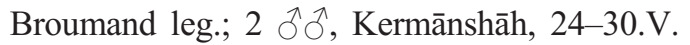
1975, M. Ghaziof leg.; 2 oq, Hormozgān (Bandar Abbās, Hajiābād), 1,050 m a.s.l., 3.V.1996, M. Badii, M. J. Ardeh \& V. Nazari leg.; 1 \%, Albors Mts (Damāvand, Kandovān), 2,000 m a.s.1., 1.VIII.2002, Dantchenko leg; 5

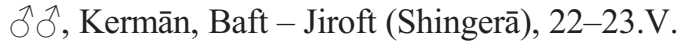
2004, H. Rajai leg.

Records from Iran. 1 đ̂, Fārs (Shirāz-Kāzerun road, Sineh Sefid), 2,200 m a.s.1., 5.VI.1937, Brandt Leg,; 2 우, Alborz (Karaj), 1936, Brandt leg.; Alborz Mts., southern parts, 30 km N Tehrān (before Shemshak), 1,800 m a.s.1., 26.(27.)V. 1971, Holzschuh leg.; Fārs (Shirāz), 10-13.V. 1974, Holzschuh leg.; Alborz Mts (Tehrān, 
Arangeh, $25 \mathrm{~km} \mathrm{~N}$ Karaj), 1,500 m a.s.1., 1-6.VI.1972, Ebert \& Falkner leg., 9.VII.1950, ca. 11,000 ft (ca. 3,350 m) a.s.l.; S W Iran, N Fārs (Barm-e Firuz), E. P. Wiltshire leg.; Esfahān, (Kāshān), 2.VI.1970, (Karkas Mt.), M. Abai leg.; Tehrān - Ghom road, (Salt lake), 70 km S Teheran, 800 m a.s.1., 6.VII.1970, M. Abai leg.; S W Iran, Fārs (Sineh Sefid), ca. 6,500ft (ca. 1,980 m) a.s.1., 19.V.1950, E. P. Wiltshire leg.; N Iran, Alborz Mts (Karaj - Chālus road), 1,650 m a.s.1., 27.VI.1969, H. G. Amsel leg.; Kohgiluyeh and Boyerahmad ( 15 km S Yāsuj), 2,050 m a.s.l., 15.VI.1972, Ebert \& Falkner leg.; S Iran, 100 km S Ābādeh, N Didegān, 2,000 m a.s.1., 9.VI.1969, H.G.Amsel leg.; Alborz Mts (Reineh- Damāvand), 2,600 m a.s.1., 21-27.VII.1936, Schwingenschuss leg.; Alborz Mts (Kandovān), 2,800 m a.s.1., 3-8.VII.1936, Schwingenschuss leg.; N Iran, Alborz Mts (Tochāl), 10,000 ft (ca. 3,050 m) a.s.1., 24.VII.1943, E. P. Wiltshire leg. (Arenberger 1981); Fārs (Sineh Sefid), Khuzestān, Yāsuj, Alvand, Gorgān, Alborz Mt., Arangheh, Nesā, Shemshak, Damāvand, Darband (Arenberger 1995b).

Distribution. Slovakia, Austria, Hungary, Croatia, Bosnia \& Herzegovina, Montenegro, Macedonia, Bulgaria, Romania, Greece, Turkey, Cyprus, Syria, Palestine, Jordan, Iran, Turkmenistan, Ukraine, Russia (Chechnya), Sardinia, Italy, France.

Remarks. The examined specimens are extremely variable in size and coloration, with a wingspan of $19-27 \mathrm{~mm}(n=34)$, while the normal range is $20-25 \mathrm{~mm}$. The wing pattern in specimens from Fars and West Azarbaijan is similar to that from eastern Anatolia, but specimens from Tehran are more or less darker with very distinct markings; two specimens from Azarbaijan (Maku) are very similar in wing pattern to those from Greece. In the male genitalia the harpe is slightly longer than that of material illustrated by Arenberger's (1995b). The specimens from Kerman have a pad with wart-shaped projections basally in the external part of both valves.

\section{Wheeleria phlomidis (Staudinger, 1870)}

Aciptilus phlomidis Staudinger, 1870, Horae Soc. Ent. Ross. 7: 282.

Material examined. $1 \hat{\sigma}$, Kohgiluyeh and
Boyerahmad (Yāsuj, Sisakht), 2,250 m a.s.l., 13.VI.1972 A. Pazuki \& G. Ebert leg.; 2 우, West Āzarbāijān (Orumieh), 1,350 m a.s.1., 7.VI.1975, M. Abai leg.; 1 †, West Āzarbāijān (Ghāsemlu), 16.VI.1975, M. Abai leg., 1 ô, West Āzarbāijān (Māku, Ārājigh, Bāshkand), 1,400 m a.s.1., 16.VI.1976, A. Pazuki \& H. Broumand leg.; 1 đ̃, Kermānshāh, 24-30.V.1975, M. Ghaziof leg., 1 q, 9.VI.1975, M. Abai leg.; 2 우, Kordestān (45 km S W Saghez), 2,000 m a.s.1., 30.VI.1975, A. Pazuki leg.; 2 đ̄ đ̄ 1 q, Tehrān (Karaj, Kandavān, Sarchāl), 2,800 m a.s.1., 4-8.VII.1977, A. Pazuki \& A. Mortazaviha leg., 1 O , Tehrān (Karaj, Āsārā), 1,850 m a.s.1., 27.VI.1971, Gh. Farahbakhsh, M. Kavian \&.M.Ghazyof leg., 1 đิ 1 ㅇ, Tehrān (Karaj, Āzādbar), 2,300 m a.s.1., 12.VII.1996, H. Barari \& M. Badii leg., Tehrān (Shemirān, Evin), 1,600 $\mathrm{m}$ a.s.1., 6.VI.1972, H. Mirzayans; 2 슈 4 우우, Māzandarān, (Baladeh, Yush), 2,100 m a.s.1., 26.VI.1998, M. Mofidi-Neyestanak leg.; 1 ふ઼, Lorestān (Khorramābād, Badrābād), 1,200 m a.s.1., 13.V.1994, A. M. Sarafrazi \& A. Hashemi leg.; 1 ふै, Lorestān (Azna, Darband), 1,700 m a.s.1., 5-6.VI.2000, M. Badii \& M. MofidiNeyestanak leg.; 1 §̄, Khuzestān (Iizeh, Āzhgil), 950 m a.s.1., 26.IV.1995, M. Parchami-Araghi, M. J. Ardeh \& M. Badii leg.; 1 ô, Ghazvin (Alamut, Kashkdasht), 1,470 m a.s.1., 19.VI. 1995, M. Parchami-Araghi, M. J. Ardeh \& E. Ebrahimi leg.; Hormozgān (Bandar Abbās, Hajiābād, 1,050 m a.s.1., 3.V.1996, M. Badii, M. J. Ardeh \& V. Nazari leg. (without abdomen); 1 ô, Fārs (Shirāz, Khānezeniān), 2,000 m a.s.l., 18.V.1974, M. Abai \& A. Pazuki leg., 1 †, Fārs (Shirāz, 10 km N Sivand), 1,150 m a.s.1., 15.V. 1974, M. Abai \& A. Pazuki leg., 1 +, Fārs (Nowdān, Gaw Koshak), 1,170 m a.s.1., 16.IV. 1975, H. Broumand leg., 2 qo 1 ふै, Fārs (Kāzerun, Nowdān), 1,250 m a.s.1., 15.IV.1975,

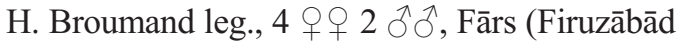
Farāshband), 7.V.4004, M. Bolonja, S. Serri \& A. Hajesmailian leg., 3 + , Fārs (Firuzābād Farāshband, Chāhturkaman), 1,200 m a.s.l.,

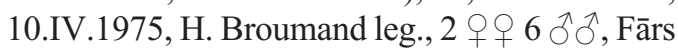
(Sārvestān, Robāt), 1,400 m a.s.1., 5.V.1996, M. Badii, M. J. Ardeh \& V. Nazari leg.

Records from Iran. 1 ô, Fārs, 28.V.1937 (5.VI.1937), (Shirāz-Kāzerun road, Sineh Sefid), 2,200 m a.s.1., Brandt leg.; 1 $\widehat{\jmath}$, Alborz 
Mts, (Karaj), 1936, Brandt leg.; NW Iran, 28 km N Sanandaj, 1,600 m a.s.1., 15.VI.1975, H. G. Amsel leg.; N Iran, Alborz Mts., (Tehrān, Arangeh), $25 \mathrm{~km} \mathrm{~N}$ Karaj, 1,550 m a.s.1., 4.VII.1972, Ebert \& Falker leg.; S Iran, Kohgiluyeh and Boyerahmad, $30 \mathrm{~km} \mathrm{~S}$ Yāsuj, Kushk, 2,200 m a.s.l., Ebert \& Falker leg.; S Iran, Fārs, 50 km NW Ardekān, (Tang-e Sorkh), 2,250 m a.s.1., 16.VI.1972, Ebert \& Falker leg.; N Iran, $40 \mathrm{~km}$ E Tehrān, 1,500 m a.s.1., 16.VI.1969, H. G. Amsel leg.; W Iran, Kordestān, (Saghez-Bāneh road, $21 \mathrm{~km}$ NE Bāneh), 1,950 m a.s.1., 30.VI-2.VII.1975, Ebert \& Falker leg.; Darband, 25 km N W Tehrān, 7-15.VI.1963, 2,000 m a.s.1., Kasy \& Vartian leg; S W Iran, E Ghasr-e Shirin, 24.V.1963, Kasy \& Vartian leg.; N Iran, 30 km W Tehrān, 2.VI.1963, Kasy \& Vartian leg. (Arenberger 1981). Alborz Mt., Chālus, Arangeh, Darband, Kordestān (Sanandaj, Bāneh), Ghasr-e Shirin (Arenberger 1995b).

Distribution. Greece, Turkey, Syria, Iran, Palestine, Jordan, Kazakhstan, Russia.

Remarks. The wingspan of the Iranian specimens is $21-30 \mathrm{~mm}(n=62)$, while the normal range is 23-28 $\mathrm{mm}$ (Arenberger 1995b).

Tabulaephorus djebeli (Arenberger, 1981)

Pterophorus djebeli Arenberger, 1981, Z. ArbGem. Öst. Ent. 32(3/4) 1980 (1981): 98.

Material examined. 1 ก̀, Golestān (Golestān National Pārk, Sulgerd), 1,100 m a.s.1., 23-24. VIII.1984, H. Broumand \& H. Myrzayans leg., 1 ふ̃, Golestān (Golestān National Pārk, Ālmeh), 1,600 m a.s.1., 19-20.VII.1985, A. Pazuki leg., 8 đ̄o 2 우, Golestān (Golestān National Pārk, Ālmeh), 1.IX.1987, A. Pazuki leg.

Records from Iran. 1 ふे, Khorāsān, Binaloud Mt. (Mashhad), 1,800 m a.s.1., 5.VIII.1938, Brandt leg. (Arenberger 1981).

Remarks. Tabulaephorus djebeli was described from several males collected by Brandt in Binalud Mt. (Mashhad, Khorasan Province in NE Iran on 5.VIII.1938); hitherto the female remained unknown. Along with the eight male specimens of $T$. djebeli from Golestān National park (Almeh), there were two females having the same collecting data as the males, which are considered to be the females of $T$. djebeli.

Description. Wingspan 20-21 mm. Head

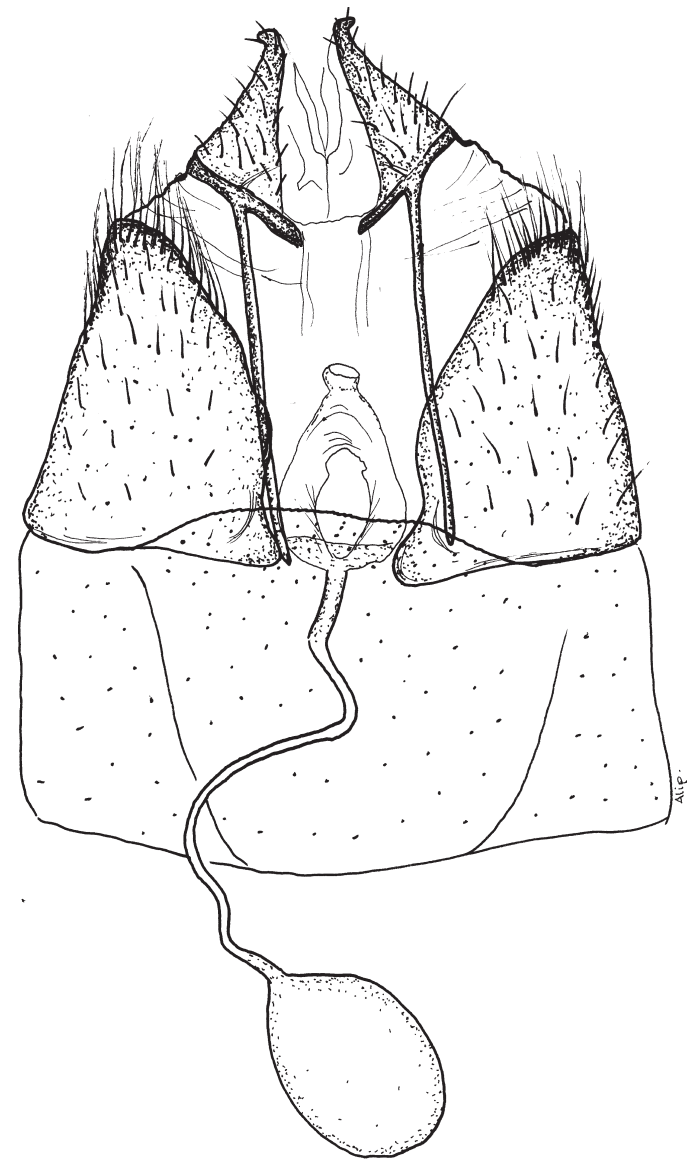

Fig.4. Female genitalia of Tabulaephorus djebeli (Arenberger).

with appressed scales, cream-whitish: collar with some erect scales; frons smoothly scaled; palps curved upward, white with rather long and erect scales on segment I (Fig. 3b). Antenna dorsally white, laterally and ventrally creamy with short cilia on ventral and lateral sides. Thorax whitish cream. Abdomen creamy white, dorsally with a longitudinal, light brown median line.

The creamy white ground colour of the forewing is very similar in both sexes. Females with some light brownish scales on the upper side of the forewing, at the base of the furcation of the first and second lobes. Fringes creamy white with a brownish area distally on the underside and a brownish area basally on the upper side of the first lobe. On the underside of the second lobe, fringes brown. Hindwings creamy white with light brown fringes.

Female genitalia (Fig. 4). Papillae anales with 
a button-shaped process distally. Ostium rounded, antrum broader than ductus bursae; ductus bursae long, almost three times longer than spherical corpus bursae; ventral plate funnel -shaped with a spherical process distally; posterior margin of sternum VII convex.

Records from Iran. Khorāsān (Binālud Mt.) (Arenberger 1995b).

Distribution. Iran.

Tabulaephorus decipiens (Lederer, 1870)

Pterophorus decipiens Lederer, 1870, Horae Soc. Ent. Ross. 8: 27 (1870)

Pterophorus tristanae Zagulajev, 1986, Trudy zool. Inst., Leningr. 67: 93-94.

Material examined. 1 ô, Tehrān (Disin), 17.VIII.1973, G. Ebert leg.; 1 đ̃, Golestān (Gorgān, Shāhkuh-e Bālā), 2,400 m a.s.1., 9.VII.2003, H. Alipanah \& E. Ebrahimi leg.; 2 ठิત, W. Alborz, Māsuleh, 2,000 m a.s.1., 3-4.VIII.2002, Dantchenko leg.

Records from Iran. Hājiābād (Arenberger 1995b).

Distribution. Iran, Russia, Armenia, Kyrgyzstan.

Remarks. The Shahkuh specimen is similar in colour and wing pattern to those illustrated by Arenberger (1995b), but the specimen from Disin is a paler light brown, without any markings on the costal margin of the forewing. In the male genitalia, the left valve is not as broad as that illustrated by Arenberger (1995b). Inner projection of tip of left valve in the examined specimens longer.

Tabulaephorus parthicus (Lederer, 1870)

Pterophorus parthicus Lederer, 1870, Horae Soc. Ent. Ross. 8: 27 (1870).

Material examined. 1 ô, Kermān (Bāft, Dareh Pahn), 1,750 m a.s.1., 21.V.1977, M. Safavi, A. Pazuki \& M. Abai leg., 1 , Kermān (Jiroft, Esfandagheh, Sargaz), 1,650 m a.s.l., 20.V.1977, A. Pazuki \&, M. Safavi leg.; 4 ふ઼ે, Fārs (Dehbid), 2,150 m a.s.1., 19.VI.1986, H. Mirzayans \& A. Hashemi leg.; 2 đ̂̉, Golestān (Golestān National Pārk, Ālmeh), 1,600 m a.s.l., 19-20.VII.1985, A. Pazuki leg.; 1 đ̂ 2 우, Chāhārmahāl and Bakhtiāri (S Shalamzār,
Tang-e Chogān), 220 m a.s.1., 11.VII.1982, H. Broumand \& A. Pazuki leg.; 3 우 2 ઈิోَ, Markazi (N E Narāgh, Gorān Mt.), 2,500 m a.s.1., 6.VII.2003, G. Ebert \& R. Trusch leg.; 9 ふ઼ 우, Esfahān (N Targh, Karkas Mt.), 2,600 m a.s.1., 8.VII.2003, G. Ebert \& R. Trusch leg.

Records from Iran. 1 ^ 1 \%, Alborz, (Karaj), 1936, Brandt leg.; 3 ふ઼ 1 †, Fārs (Shirāz-Kāzerun road, Sineh Sefid), 2,200 m a.s.1., 1937 (Bigot 1968); Gorgān (Astrābād), Haberhauer leg.; Fārs, (Ardekān-Tol-e Khosro road, Komehr), 2,600 m a.s.1., Brandt leg. (GU 4093 q NRS); S Didegān, 160 km N Shirāz, 1,900m a.s.1., 8.VI.1969, H. G. Amsel leg.; Fārs, Ābādeh, 1,550 m a.s.1., 4.VII.1972, Ebert \& Falkner leg. (Arenberger 1981); Gorgān, Hājiābād, Alborz Mt., Karaj, Arangeh, Ābādeh, Didegān, Kāzerun, Shāhkuh Mt. (Arenberger 1995b).

Distribution. Ponto-Mediterranean; known from Turkey, Syria, Palestine, Jordan, Iran and Afghanistan.

Remarks. The examined specimens are similar to those illustrated by Arenberger (1995b), but one from the Karkas Mountain is smaller in size (wingspan $17.5 \mathrm{~mm}$ ). In the female genitalia, the shape of the papillae anales of the examined specimens is totally different from those illustrated by Arenberger's (1995b) and very similar to Tabulaephorus hissaricus (Zagulajev, 1986), but with a longer ventral plate.

\section{Calyciphora golestanica sp. $n$.}

Type material. Holotype đ̊̄, Iran, Golestān province, Golestān National Pārk, Ālmeh, 1,650 m a.s.1., 17-25.V.1988, A. Pazuki leg., Genitalia slide, HA-23LP-HM (HMIM, PPDRI), Paratypes: 2 ठิ 1 \%, Iran, Golestān province, Golestān National Pārk, Sulgerd, 1,150 m a.s.1., \& 7.V.1999, M. Moghaddam, S. Manzari \& H. Barari leg. Holotype deposited at the Hayk Mirzayans Insect Museum.

Description. Male and female. Wingspan 19-21 mm. Head smooth scaled, brown: collar with some erect creamy scales; frons smooth, sparsely scaled. Palpi curved upward, as long as diameter of eye, with some long, erect, creamy white scales at base of segment I; scales of other segments smooth, brownish. Antennae ringed, with cap-shaped scales dorsally at base (Fig. 3c); 


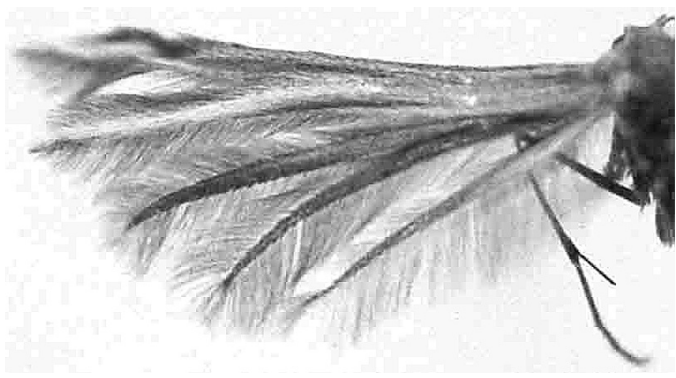

Fig.5. Calyciphora golestanica sp. n.

dorsally white and ventrally brownish; shortly ciliated on ventral side. Thorax creamy brown. Abdomen dirty cream, without any markings dorsally. Hindlegs covered with creamy and chocolate-brown scales. Spurs of the same pair of unequal length.

Forewing (Fig. 5). Basally greyish chocolate-cream, mixed with whitish scales at first and second lobes and a narrow white line at dorsum; fringes in upper and lower sides of first and second lobes dark brown, without any markings. Underside of forewing light brown except for white area in first and second lobes.

Hindwing (Fig. 5). First and second lobes brown. Third lobe at costal half brown and at dorsal half creamy white, with some long hairs at dorsum. Underside of first and third lobes creamy

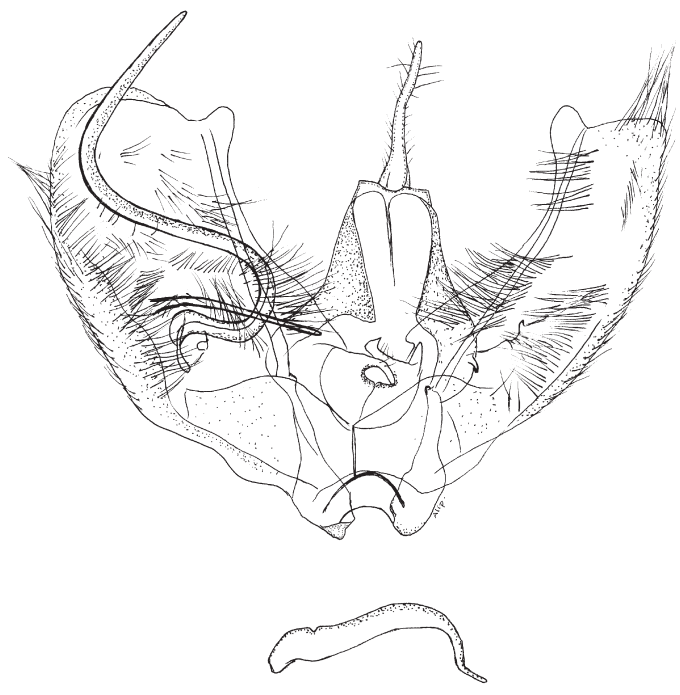

Fig.6. Male genitalia of Calyciphora golestanica sp. n. (holotype).

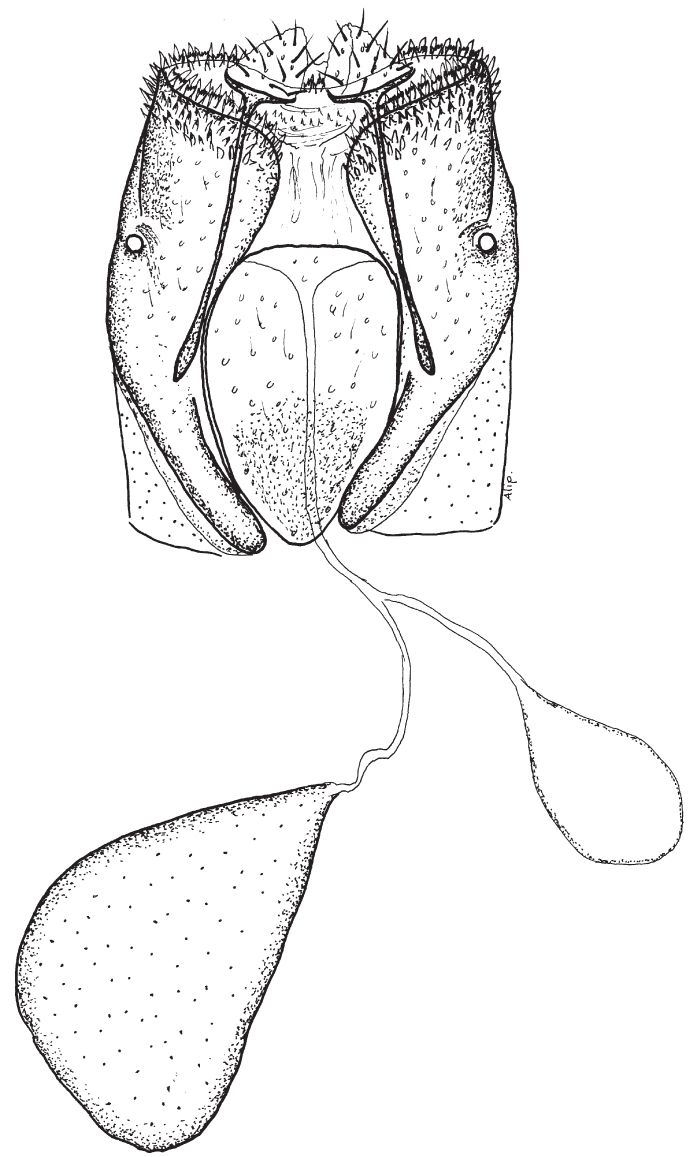

Fig.7. Female genitalia of Calyciphora golestanica sp. n. (paratype).

white, that of second lobe brownish.

Male genitalia (Fig. 6). Genitalia asymmetrical. Left valve broader than right one; both valves with a process on upper border of costa; left valva with a long, S-shaped harpe extending out of valva and a thorn-like process near harpe, directed downward obliquely; right valva without saccular process; sacculus in both valves similar; uncus long, more than 1/3 length of valva; tegumen with pointed edges; aedeagus narrow, straight basally, terminal half S-shaped.

Female genitalia (Fig. 7). Ventral plate longer than broad; lateral structures long with blunt apex, both with a hole medially; ostium broad, antrum not funnel-shaped as in $C$. albodactylus. Ductus bursae narrow, elongate and slender; vesica seminalis vesicular, the extension of ductus seminalis closer to ventral plate than corpus 
bursae; corpus bursae pear-shaped. Anterior apophyses absent.

Diagnosis. In the male genitalia the new species is close to C. marashella Zagulajev, 1986 from which it differs by the following characters. In the male genitalia, the lobe-like process on the left valva looks like a small knob set perpendicularly to the valva, while in C. marashella it is slanting. The lobe-like process of the right valva is shorter than that of C. marashella. The harpe on the left valva is strongly bent distally, but straight in C. marashella.

The female genitalia of the new species are also similar to those of $C$. marashella, but differ in the following characteristics: Antrum broad, not so funnel-like as in C. marashella; lateral processes of ventral plate longer and narrower than those of C. marashella; ductus seminalis arising from middle of the ductus, not from the base of the corpus bursae as in C. marashella.

Variation. There is no significant variation in the examined specimens.

Distribution. Iran.

\section{Calyciphora albodactylus (Fabricius, 1794)}

Pterophorus albodactylus Fabricius, 1794: 348. Pterophorus xerodactyla Zeller, 1841: 860. Pterophorus xanthodactylus auct. (nec Treitschke, 1833).

Aciptilia xanthodactyla var. sicula Fuchs, 1901: 72.

Records from Iran. Iran; Fazekas (1993).

Distribution. Spain, France, Sicily, Italy, Switzerland, Belgium, Germany, Sweden, Finland, Slovakia, Austria, Hungary, Croatia, Macedonia, Bosnia \& Herzegovina, Bulgaria, Romania, Greece, Turkey, Iran, Ukraine, Russia (Chechnya).

\section{Calyciphora nephelodactyla (Eversmann,} 1844)

Alucita nephelodactyla Eversmann, 1844, Fauna Lepid. Volgo-Ural.: 609.

Pterophorus subalternans Lederer, 1870, Horae Soc. Ent. Ross. 6: 93.

Aciptilia apollina Milličre, 1882, Lépidoptčrologie [2] (8): 25, 36.

Calyciphora extensa Zagulajev, 1986, Trudy zool. Inst., Leningr. 4: 180.

Records from Iran. Hyrc.(ania), as Alucita subalternans Lederer, 1870 (Staudinger \& Rebel 1901).

Distribution. Spain, France, Italy, Switzerland, Austria, Hungary, Slovakia, Poland, Bosnia \& Herzegovina, Macedonia, Bulgaria, Greece, Turkey, Syria, Russia, Georgia, Iran.

\subsection{Tribus Oidaematophorini}

Puerphorus olbiadactylus (Millière, 1859)

Pterophorus olbiadactylus Milličre, 1859, Iconogr. Descr. Chenilles Lépid. Inédits 1: 89-91.

Pselnophorus hemigargus Meyrick, 1908, Trans. Ent. Soc. Lond. 1907 (1908): 491.

Gypsochares leptodactyla Staudinger (Caradja, 1920) (nomen nudum).

Records from Iran. Fārs (Sineh Sefid) (Arenberger 1995b).

Distribution. Mediterranean region eastward to Central Asia.

\section{Pselnophorus poggei (Mann, 1862)}

Oxyptilus brachydactylus var. poggei Mann, 1862, Wien. Ent. Monatsschr. 6: 409.

Leioptilus minutus Alpheraki, 1876, Trudy russk. Ent. Obshch. 10: 32-33.

Pselnophorus borzhomi Zagulajev, 1987, Ent. Obozr. 66 (2): 363-365.

Records from Iran. Māzandarān, Darband, Gorgān (Arenberger 1995b).

Distribution. Russia, Ukraine, Turkey, Georgia, Iran.

Emmelina monodactyla (Linnaeus, 1758)

Phalaena monodactyla Linnaeus, 1758, Syst. Nat. (Edn 10) 1: 542.

Phalaena bidactyla Hochenwarth, 1758, Schrift. Berl. Ges. Naturf. Fr. 6: 336.

Alucita pterodactyla Hubner, (1805) (nec Linnaeus, 1758), Samml..eur. Schmett. 9

Pterophorus flaveodactylus Amary, 1840, Esercit. Accad. Aspir. Nat. Napoli 2 (1): 84.

Pterophorus pterodactylus Zeller, 1841 (nec 
Linnaeus, 1758), Isis Oken, Leipzig 1841: 846-848.

Pterophorus cineridactylus Fitch, 1854, Trans. N. Y. Agr. Soc. 14: 848

Pterophorus naevosidactulus Fitch, 1854, Trans. N. Y. Agr. Soc. 14: 849.

Pterophorus impersonalis Walker, 1864, Cat. BM, 942.

Pterophorus pergracilidactylus Packard, 1873, Ann. Lyc. Nat. Hist.N.Y. 10: 266.

Pterophorus barberi Dyar, 1903, Proc. Ent. Soc. Wash. 5: 228.

Pterophorus pictipennis Grinnel, 1908, Canad. Ent. 40 (9).

Pterophorus monodactylus $f$. rufa Dufrane, 1960, Bull. R. Inst. Sci. nat. Belg. 36 (29).

Material examined. 1 , Khuzestān (Dezful, Safiābād), 150 m a.s.1., 23.IV.1995, M. Parchami Araghi, M. J. Ardeh \& M. Badii leg.; 1 q, West Āzarbāijān (Khoy, Habasheh-e Soflā), 1,825 m a.s.1., 19-20.VIII.1994, A. M. Sarafrazi \& E. Ebrahimi leg.; 1 đ̃, West Āzarbāijān (Rājān, 30 km S W Orumieh), 1,650 m a.s.1., 24.VII.1976, A. Pazuki \& H. Broumand leg.; 1 q, East Āzarbāijān (Kaleibar, Vināgh), 800 m a.s.1., 7-8.VIII.1992, M. Parchami Araghi \& M. Badii leg.; 2 đิ̊̄, East Āzarbāijān (Tabriz, Kalātpushān), 26.VIII.1974, Mashayekhi leg.; 1 , Zanjān (Soltānieh, Asadābād), 1,750 m a.s.1., 14.VIII.1993, E. Ebrahimi \& A. M. Sarafrazi leg.; 1 †, Tehrān (Varamin), 14.VII.1949, S. Eghlidi leg., 1 ô, (Karaj, Kalhā), 2,000 m a.s.1., 25-26.VIII.1996, H. Barari, M. Badii \& A. M. Sarafrazi leg., 1 ふૈ, (Karaj), 29.IX.1964, H. Javan-Moghaddam leg.; 1 q, Khorāsān (Mashhad, Torogh), 23.VII.1973, Zare leg., 1 (Kāshmar), 24.VII.1971, A. Pazuki \& M. Ayatollahi leg.; 1 đ̊ 1 †, Semnān (Shāhrud, S S Shāvār Mt.), 2,030 m a.s.1., 26-28.VIII.1983, H. Broumand \& A. Pazuki leg.; 1 + , Māzandarān (Sāri), 0 m a.s.1., 23.VI.1995, H. Mirzayans, A. M. Sarafrazi \& M. Badii leg., 1 \&, Māzandarān (Tonekābon, Sehezār, Āsh Mahaleh), 750 m a.s.1., 7.IX.1990, E. Ebrahimi \& M. Badii leg.; 2 đ̃ô 1 †, Golestān (Golestān National Pārk, Tang-e Gol), $620 \mathrm{~m}$ a.s.1., 23-25.V.1986, A. Pazuki leg., 2 đิ 1 1 , Golestān (Golestān National Pārk, Tang-e Gol), 720 m a.s.l., 29-30.XI. 1998, S. Manzari, M. Moghaddam \& N. Nazari leg., 1 ô, Golestān (Golestān National Pārk,
Tang-e Gol), 5.V.1999, M. Moghaddam, H. Barari \& S. Manzari leg., 1 đ̄, Golestān (Golestān National Pārk, Kārkandeh), 5.VII. 2003, H. Rajai leg., 1 đิ 2 우, Golestān (Gorgān, Shastkolā), $500 \mathrm{~m}$ a.s.1., 28.VII.1996, E. Ebrahimi \& V. Nazari leg.; 1 ㅇ, Gilān (Lowshān, Bivarzin), 900 m a.s.1., 27-28.V.1997, M. Badii, V. Nazari \& A. M. Sarafrazi leg., 1 đ̊, Gilān (Bandar-e Anzali, 15 km Asālem-Pissāson), 220 m a.s.1., 30.VIII.1975, H. Mirzayans leg., 1 q, Gilān (Asālem, Sheikh mahal), 160 m a.s.1., 28-30.VI.1977, A. Pazuki \& M. Mortazaviha leg., 2 đ̄ đ̂ 1 ๆ, Gilān (Asālem, Pisāson), 1,300 m a.s.1., 15-16.VIII.1980, A. Pazuki \& H. Broumand leg., 1 \&, Gilān (Asālem), 29.IX.1970, M. Abai leg., 1 đ̃, Gilān (Hashtpar, Rek), $570 \mathrm{~m}$ a.s.1., 31.VIII.1975, H. Mirzayans leg.; 1 ㅇ, Hamedān (Hamedān, Darreh-e Morādbeik), 2,200 m a.s.1., 25-26.VIII. 1995, H. Mirzayans \& M. Badii leg.; 1 q, Lorestān (Khorramābād, Manuchehrābād), 1,150 m a.s.1., 4.V.1994, A. Hashemi \& A. M. Sarafrazi leg., 1 †, Lorestān (Aznā, Oshtorānkuh, Kamandān), 2,050 m a.s.1., 23.VIII.1995, H. Mirzayans \& M. Badii leg.; 1 đ̄, Esfahān (35 km $\mathrm{N}$ Meymeh), 2,050 m a.s.1., 6.VIII.1978, A Pazuki \& H. Broumand leg., 1 đิ 1 ㅇ, Esfahān (Kāshān, Niāsar, Sericheh), 1,650 m a.s.l., 29-31.VII.1983, A Pazuki \& A. Hashemi leg.; 1 ㅇ, Ghom (Vesb), 2,300 m a.s.1., 5.VIII.1988, A. Hashemi leg.; 2 우, Kermān (Jiroft, Narāb), 900 m a.s.1., 16.XI.1999, M. Badii, H. Barari \& M. Mofidi-Neyestanak leg.; 1 q, Alborz Mts, Damāvand, Kandovān, 2,000 m a.s.l., 1.VIII.2002, Dantchenko leg.

Records from Iran. 1 ô, Tār lake area (2,100-2,200m a.s.1.), 14-17.VII. (Zerny, 1940); 1 đิ 1 ㅇ, Tāhergurābe, 20.X.1954 (Amsel 1959); 2 ऽ઼ 7 우, Alborz, (Karaj), V-VI.1936 (Bigot 1968); Rasht, Chālus, Tahergurab, Hājiābād (Arenberger 1995b).

Distribution. Europe (excluding Arctic regions), North Africa, Central Asia, North America.

Remarks. Forewing varies from cream and ochreous to light brownish grey and grey.

Emmelina argoteles (Meyrick, 1922)

Pterophorus algoteles Meyrick, 1922, Exot. Microlepid. 2: 549, 550. 
Pterophorus jezonicus Matsumura, 1931, I11. Ins. Japan: 1057, No. 2076.

Pterophorus komabensis Matsumura, 1931, I11. Ins. Japan: 1057, No. 2077.

Pterophorus menoko Matsumura, 1931, I11. Ins. Japan: 1057, No. 2079.

Pterophorus yanagawanus Matsumura, 1931, I11. Ins. Japan: 1058, No. 2080.

Emmelina jezonica pseudojezonica Derra, 1987, Nota Lepid. 10 (1): 71-78.

Material examined. 1 đ̊, Fārs (Kāzerun), 970 m a.s.1., 10.X.1994, M. Abai leg.; 1 q, Māzāndarān (Nowshahr, Nāyrank), 0 m a.s.l., 10.X.1995, A. Rezwani, M. Badii \& E. Ebrahimi leg.

Distribution. France, Croatia, Italy, Spain, Switzerland, Germany, Austria, Slovakia, Hungary, Bulgaria, Russia, Georgia, India, China, Japan.

Remarks. In the male genitalia of the examined specimen, the membranous projection of the left valva is slender, digitate at half its length, while the remaining half is broad (cf. Arenberger $1995 b)$. On the left valva a small projection is present in its distal part (Fig. 2d). New to the fauna of Iran.

Adaina microdactyla [Hübner, (1813)]

Alucita microdactyla Hübner, [1813] 1796, Samml. Eur. Schmett. 9.

Pterophorus carphodactylus Stephens, 1834 [nec Hübner, (1813)], I11. Brit. Ent. (Haustellata) 4: 374.

Adania montivola Meyrick, 1937, Dt. Ent. Z. Iris 51: 170.

Records from Iran. 1 \ 2 우, Alborz, (Karaj), VIII-IX.1936, 1 ふ̄, Fārs (Ardekān-Tol-e Khosro road, Komehr), 4.VII.1937 (Bigot 1968); Karaj (Alborz Mt.), Fārs, Hājiābād, Gorgān (Arenberger 1995b).

Distribution. England, France, Spain, Portugal, Morocco, Italy, Corsica, Sardinia, Switzerland, Germany, Belgium, The Netherlands, Denmark, Sweden, Finland, Lettland, Poland, Slovakia, Austria, Hungary, Slovenia, Croatia, Bulgaria, Romania, Greece, Turkey, Palestine, Iran, Georgia, Russia (Chechnya), Nepal, China, Taiwan, Japan.
Hellinsia lienigianus (Zeller, 1852)

Pterophorus lienigianus Zeller, 1852, Linn. Ent. 6: $380,381$.

Pterophorus melinodactylus Herrich-Schäffer, 1855, Syst. Bearb. Schmett. Eur. 5.

Pterophorus scarodactylus Becker, 1861, [nec Hubner (1813)], Annls Soc. Ent. Belg. 5: 56-57.

Leioptilus serindibanus Moore, 1886, Lepid. Ceylon 3: 527.

Leioptilus sericeodactylus Pagenstecher, 1900, Zoologica 29: 240.

Ovendenia septodactyla sensu Tutt, 1905 nec Alucita septodactyla Treitschke, 1833.

Pterophorus victorianus Strand, 1913, Arch. Naturgesch. 78: 130.

Pterophorus lienigianus var. catharodactylus Caradja, 1920 (partim), Dt., Ent. Z. Iris 34: 86.

Pterophorus hirosakianus Matsumura, 1931, 6000 I11. Ins. Japan: 1056.

Oidaematophorus mutuurai Yano, 1936, Pacific Insects 5 (1): 180-182.

Records from Iran. 1 +, Alborz, Karaj, 27.V.1936 (Bigot 1968).

Distribution. Transpalaearctic: from England to Japan, excluding the Arctic regions and the Iberian Peninsula.

Hellinsia pectodactyla (Staudinger, 1859)

Pterophorus pectodactylus Staudinger, 1859, Dts. Ent. Ztschr. Iris 20: 258.

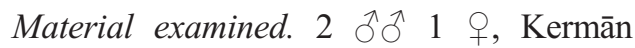
(Rāyen, Kuh-e Hezār), 2,600-2,800 m a.s.1., 26.V.2004, H. Rajai leg.

Distribution. Europe, Kazakhstan, Afghanistan, India.

Remarks. New to the fauna of Iran.

Oidaematophorus lithodactylus (Treitschke, 1833)

Alucita lithodactyla Treitschke, 1833, [in] Ochsenheimer, Schmett. Eur. 9 (2): 245.

Alucita septodactyla Treitschke, 1833, [in] Ochsenheimer, Schmett. Eur. 9 (2): 246.

Pterophorus similidactylus Dale, 1834, Mag. Nat. Hist. J. 7: 263.

Pterophorus phaeodactylus Stephens, 1834 
[nec Alucita phaeodactyla Hübner, (1813)], I11. Brit. Ent. (Haustella) 4: 375.

Pterophorus lithoxylodactylus Duponchel, (1840), Hist. Nat. Lépid. 11: 670.

Records from Iran. Marivān; Arenberger (1995b).

Distribution. Widely distributed in the Palaearctic region.

Acknowledgements. We thank Dr. Cees Gielis (National Natuurhistorisch Museum: Naturalis, Leiden, The Netherlands) for his important and helpful guidance. We acknowledge Mr. Mohsen Mofidi-Neyestanak (Plant Pests and Diseases Research Institute) for his general help. We are grateful to Dr. Oleg Kosterin, Dr. S. Manzari (Plant Pests and Diseases Research Institute) for critically reading the manuscript, Dr. W. Gerald Tremewan for his valuable comments on the manuscript (Natural History Museum, London) and to Mrs. Parsi for drawing one of the illustrations.

\section{References}

Amsel, H.G. 1959: Microlepidoptera aus Iran. - Stuttg. Beitr. Naturk. 28: 29-30.

Arenberger, E. 1981: Die Pterophorus-Arten West- und Zentralasiens. 1. Beitrag. Z. ArbGem. — Ost. Ent. 32: 97-110.

Arenberger, E. 1995a: Die Pterophoridae der osterreichischen Turkmenistan-Expedition 1993. Z. ArbGem. - Ost. Ent. 47: 55-58.

Arenberger, E. 1995b: Pterophoridae. - In: H. G. Amsel, F. Gregor and H. Reisser (eds.), Microlepidoptera Palaearctica. Vol. 9. George Fromme, Vienna. $\mathrm{XXV}+258 \mathrm{pp}+153 \mathrm{pls}$.
Arenberger, E. 2002: Eine neue Agdistis aus Zentralasien (Lepidoptera, Pterophoridae). — Carinthia. 192/112: 607-614.

Bigot, L. 1968: Les Pterophoridae de l'Iran (Lep.). Arkiv for Zoologi, Band 20, Nr. 12 (8): 243-251.

Gielis, C. 1996: Microlepidoptera of Europe, vol.1: Pterophoridae. - Apollo Books, Stenstrup. 222 pp.

Gielis, C. 2003: World Catalogue of Insects. 4: Pterophoroidea \& Alucitoidea. - Apollo Books, Stenstrup.

Fazekas, I. 1993: Beitrage zur Kenntnis der Pterophoridae-Fauna Ungarns, Nr. 2. Die Federmotten Nord-Ungarns (Nordliches Mittelgebirge) Lepidoptera: Pterophoridae. - Folia Historico Naturalia Musei Matraensis 18: 97-137.

Kallies, A. \& Spatenka, K. 2003: The Clearwing Moths of Iran (Lepidoptera, Sesiidae) (part 1). — Linneanna Belgica, Pars XIX, N. 2: 81-126.

Karlshot, O. \& Gielis, C. 1995: The Pterophoridae described by J. C. Fabricius, with remarks on type material of Fabrician Lepidoptera (Insecta). — Steenstrupia 21: 31-35.

Lederer, J. 1869: Verzeichniss der von Herrn Jos. Haberhauer dei Astrabad in Persien gesammelten Schmetterlinge. - Horae Soc. Ent. Ross. 6: 6-93.

Lederer, J. 1871 (1870): Nachtrag zum Verzeichnisse der von Herrn Jos. Haberhauer dei Astrabad in Persien gesammelten Schmetterlinge. - Horae Soc. Ent. Ross. 8: 3-28.

Staudinger, O. \& Rebel, H. 1901: Katalog der Lepidopteren des palaarktischen Faunengebietes. Berlin. I - 441, II - 1-368.

Toll, S. 1947: Beitrag zur Mikrolepidopterenfauna von Nordost-Persien. - Z. Wien. Ent. Ges. 32: 107-116.

Zagulajev, A. K. 1986: A guide to the insects of the European part of the USSR, Lepidoptera. Family Alucitidae. - Opredelitel Faune SSR 43 (144): 215-228.

Zerny, H. 1940: Mikrolepidopteren aus dem ElbursGebirge in Nord-Iran. Z. Wien. - Ent. Ver. 25: 22. 